\title{
Jean Mawhin's contributions to critical point theory
}

\section{Michel Willem*}

Dedicated to Jean Mawhin on the occasion of his seventieth birthday with friendship.

${ }^{\text {*Correspondence: }}$

Michel.Willem@uclouvain.be

Institut de Recherche en

Mathématique et Physique,

L7.01.02, Chemin du Cyclotron 2,

Louvain-la-Neuve, 1348, Belgium

\section{Abstract}

The aim of this article is to describe some fundamental contributions of Jean Mawhin to critical point theory and its applications to boundary value problems.

\section{Content}

The first paper by Jean Mawhin on critical point theory [1] was published in 1982 and was devoted to periodic solutions of a forced pendulum equation. One of the most recent papers in 2012 [2] concerns periodic solutions of difference systems with $\phi$-Laplacian. It is impossible to describe all the contributions. We have selected 17 articles, 2 books and some fundamental topics:

- the forced pendulum equation,

- convex perturbations of indefinite quadratic functionals,

- construction of almost critical points,

- converse to the Lagrange-Dirichlet theorem, and

- Neumann problems for the $\phi$-Laplacian.

\section{From the classical to the relativistic pendulum}

The forced pendulum equation is an important field of investigations of Jean Mawhin. We describe only some contributions (by variational methods) to the conservative forced pendulum, and we refer to the exhaustive survey [3] for other results.

Consider the classical second-order problem:

$$
\left\{\begin{array}{l}
u^{\prime \prime}=f(u)+h(t), \\
u(0)=u(T), \quad u^{\prime}(0)=u^{\prime}(T),
\end{array}\right.
$$

where $f$ is $2 \pi$-periodic. The solutions of (1) are the critical points of the action functional

$$
\Psi: H_{T}^{1} \rightarrow \mathbb{R}: u \mapsto \int_{0}^{T}\left(\frac{u^{\prime 2}}{2}+F(u)+h(t) u\right) d t,
$$

( 2013 Willem; licensee Springer. This is an Open Access article distributed under the terms of the Creative Commons Attribution License (http://creativecommons.org/licenses/by/2.0), which permits unrestricted use, distribution, and reproduction in any medium, provided the original work is properly cited. 
where $F(r)=\int_{0}^{r} f(s) d s$. The space $H_{T}^{1}$ is the space of absolutely continuous functions $u$ which are $T$-periodic and such that $\dot{u} \in L_{\text {loc }}^{2}(\mathbb{R})$. Assuming that

$$
\int_{0}^{2 \pi} f(s) d s=0, \quad \int_{0}^{T} h(t) d t=0
$$

it is not difficult to prove that $\Psi$ achieves its infimum on $H_{T}^{1}$ and, consequently, that (1) is solvable. This result, due essentially to Hamel in 1922, was rediscovered by Willem in 1981 and by Dancer in 1982.

Some sixty years after the first one, a second periodic solution was discovered in [4] under assumption (2).

Since, by assumption (2),

$$
\Psi(u+2 \pi)=\Psi(u),
$$

a natural space of definition for $\Psi$ is

$$
X=\mathbb{S}^{1} \times \widetilde{H}_{T}^{1}=\mathbb{S}^{1} \times\left\{u \in H_{T}^{1}: \int_{0}^{T} u d t=0\right\} .
$$

The functional $\Psi$ is bounded from below on $X$ and, by a category argument, has at least two geometrically distinct critical points. A generalization to systems is contained in [5].

The argument in [4] was to use a refinement of the mountain pass theorem, observing that if $v$ is a minimizer of $\Psi$, then, for all $0<r<2 \pi T$,

$$
\Psi(v) \leq \inf _{\|u-v\|_{T}^{1}=r} \Psi(u) .
$$

Another proof, using a generalization of the Poincaré-Birkhoff theorem, was suggested by Franks [6]. However, this proof is not complete [7]. It seems that the variational proof is the only one until now. To find a proof using a fixed point theorem is an interesting challenge. Moreover, there is no exhaustive description of the set of $h$ such that (1) is solvable assuming that $f$ is $2 \pi$-periodic and $\int_{0}^{2 \pi} f(s) d s=0$ (see [3] and [8]).

Let us recall the general notion of $\phi$-Laplacian. Let $\phi: \mathbb{R} \rightarrow \mathbb{R}$ (classical), or $\phi: \mathbb{R} \rightarrow$ ]- $a, a[$ (bounded), or $\phi:]-a, a[\rightarrow \mathbb{R}$ (singular) be an increasing homeomorphism such that $\phi(0)=0$. Canonical examples are, respectively, as follows:

$$
\begin{aligned}
& \phi(s)=|s|^{p-2} s \quad(p \text {-Laplacian), } \\
& \phi(s)=\frac{s}{\sqrt{1+s^{2}}} \quad \text { (curvature), } \\
& \phi(s)=\frac{s}{\sqrt{1-s^{2}}} \quad \text { (special relativity). }
\end{aligned}
$$

The case of the $p$-Laplacian for the problem

$$
\left\{\begin{array}{l}
\left(\phi\left(u^{\prime}\right)\right)^{\prime}=f(u)+h(t), \\
u(0)=u(T), \quad u^{\prime}(0)=u^{\prime}(T)
\end{array}\right.
$$

was recently solved by Jean Mawhin in [9]. The results are similar to the classical pendulum. 
Consider now the forced relativistic pendulum and assume that

$$
\left\{\begin{array}{l}
\Phi \in \mathcal{C}([-a, a]) \cap \mathcal{C}^{1}(]-a, a[), \\
\left.\phi=\Phi^{\prime}:\right]-a, a[\rightarrow \mathbb{R} \text { is an increasing homeomorphism such that } \phi(0)=0 .
\end{array}\right.
$$

On

$$
C=\left\{u: \mathbb{R} \rightarrow \mathbb{R}: T \text {-periodic such that }[[u]]=\sup _{x, y \in \mathbb{R}} \frac{|u(x)-u(y)|}{|x-y|} \leq a\right\},
$$

we define the action

$$
\Psi(u)=\int_{0}^{T}\left(\Phi\left(u^{\prime}\right)+F(u)+h(t) u\right) d t
$$

Let us describe the recent results (2010) of Mawhin and Brezis on the relativistic pendulum [10]. We sum up the simple and beautiful proof.

Theorem 1.1 Under assumptions (2) and (4), problem (3) has a solution which minimizes $\Psi$ on $C$.

Lemma 1.2 The action $\Psi$ has a minimizer on $C$.

Proof Let $\left(u_{n}\right) \subset C$ be such that $\Psi\left(u_{n}\right) \rightarrow \inf _{C} \Psi$. Since $\Psi(v+2 \pi) \equiv \Psi(v)$, we can assume that

$$
0 \leq \bar{u}_{n}=\frac{1}{T} \int_{0}^{T} u_{n} d t<2 \pi
$$

It is clear that $\sup _{n}\left\|u_{n}\right\|_{\infty}<\infty$ since $\left[\left[u_{n}\right]\right] \leq a$. By going if necessary to a subsequence, we can assume that $u_{n} \rightarrow u$ uniformly on $\mathbb{R}$ and $[[u]] \leq a$. It remains only to prove that

$$
\int_{0}^{T} \Phi\left(u^{\prime}\right) d t \leq \varliminf_{n \rightarrow \infty} \int_{0}^{T} \Phi\left(u_{n}^{\prime}\right) d t
$$

For any $0<\lambda<1$, we have that

$$
\int_{0}^{T} \Phi\left(\lambda u^{\prime}\right) d t+\int_{0}^{T} \phi\left(\lambda u^{\prime}\right)\left(u_{n}^{\prime}-\lambda u^{\prime}\right) d t \leq \int_{0}^{T} \Phi\left(u_{n}^{\prime}\right) d t
$$

so that

$$
\int_{0}^{T} \Phi\left(\lambda u^{\prime}\right) d t \leq \int_{0}^{T} \Phi\left(\lambda u^{\prime}\right) d t+(1-\lambda) \int_{0}^{T} \phi\left(\lambda u^{\prime}\right) u^{\prime} d t \leq \varliminf_{n \rightarrow \infty} \int_{0}^{T} \Phi\left(u_{n}\right) d t .
$$

We conclude by letting $\lambda \uparrow 1$.

Let us recall the notion of critical point in the sense of Szulkin [11]. 
Definition 1.3 Let $X$ be a Banach space and let $I=J+K$, where $J \in \mathcal{C}^{1}(X, \mathbb{R})$ and $K: X \rightarrow$ ]$-\infty,+\infty$ ] is convex, proper (i.e., $K \not \equiv+\infty$ ) and lower semi-continuous (l.s.c. in short). A point $u \in X$ is a critical point of $I$ if $K(u)<\infty$ and, for all $v \in X$,

$$
0 \leq\left(J^{\prime}(u), v-u\right)+K(v)-K(u)
$$

The easy proof of the next lemma is given in [11].

Lemma 1.4 Each local minimum of $I=J+K$ is a critical point of $I$.

We conclude the proof by using an argument due to Bereanu, Jebelean and Mawhin [12].

Proof of Theorem 1.1 Let $u$ be a minimizer of $\Psi$ on $C$. We have only to prove that $[[u]]<a$ in order to verify the Euler equation.

Let us define on

$$
X:\{v: \mathbb{R} \rightarrow \mathbb{R}: T \text {-periodic and such that }[[v]]<+\infty\}
$$

the functionals

$$
\begin{aligned}
& K(v)= \begin{cases}\int_{0}^{T} \Phi\left(v^{\prime}\right) d t, & v \in C, \\
+\infty, & v \in X \backslash C,\end{cases} \\
& J(v)=\frac{T}{2} \bar{v}^{2}+\int_{0}^{T}(f(u)+h(t)-\bar{u}) v d t .
\end{aligned}
$$

By an explicit computation, the problem

$$
\left\{\begin{array}{l}
\left(\phi\left(v^{\prime}\right)\right)^{\prime}=\bar{v}+f(u)+h(t)-\bar{u}, \\
v(0)=v(T), \quad v^{\prime}(0)=v^{\prime}(T)
\end{array}\right.
$$

has exactly one solution $w$ and $[[w]]<a$. Since $I=J+K$ is strictly convex, $w$ is the only solution of

$$
0 \leq\left(J^{\prime}(w), v-w\right)+K(v)-K(w), \quad \forall v \in K
$$

But, by Lemma 1.4, we have that

$$
0 \leq\left(J^{\prime}(u), v-u\right)+K(v)-K(u), \quad \forall v \in K .
$$

We conclude that $u=w$ and

$$
[[u]]=[[w]]<a .
$$

The case of Lagrangian systems of relativistic oscillators was recently treated by Mawhin and Brezis in [13]. 
An open problem from [10] is the extension in higher dimensions, for example,

$$
\min _{u \in C} \int_{\mathbf{T}^{N}}\left(1-\sqrt{1-|\nabla u|^{2}}+F(u)+h(x) u\right) d x
$$

where

$$
C=\left\{u \in C\left(\mathbf{T}^{N}\right): \sup _{x, y \in \mathbf{T}^{N}} \frac{|u(x)-u(y)|}{|x-y|} \leq 1\right\} .
$$

\section{Convex perturbations of indefinite quadratic functionals}

The dual least action principle of Clarke (see [14, 15]) was used in [16-19] and [20] to solve problems of the form

$$
L u=\nabla F(x, u) \quad\left(\text { with } \nabla F=D_{u} F\right)
$$

in a closed subspace $V$ of $L^{2}\left(\Omega ; \mathbb{R}^{M}\right)$, where $\Omega$ is a bounded domain of $\mathbb{R}^{N}$. The linear operator $L: D(L) \subset V \rightarrow V$ is self-adjoint and the nonlinear potential $F: \Omega \times \mathbb{R}^{M} \rightarrow \mathbb{R}$ is convex in its second variable.

It is always assumed that $F$ is dominated at infinity by $\lambda_{1}|u|^{2} / 2$, with $\lambda_{1}$ the first positive eigenvalue of $L$, and that there exists $w \in \operatorname{Ker} L$ such that

$$
\nabla F(x, w) \in(\operatorname{Ker} L)^{\perp}
$$

Let us denote by $K$ the inverse of

$$
L: D(L) \cap(\operatorname{Ker} L)^{\perp} \rightarrow(\operatorname{Ker} L)^{\perp} .
$$

The dual action is defined on $(\operatorname{Ker} L)^{\perp}$ by

$$
\Psi(v)=\int_{\Omega}\left[\frac{1}{2}(K v \mid v)+F^{*}(x, v)\right] d x,
$$

where $F^{*}(x, v)$ is the Fenchel transform of $F(x, u)$ :

$$
F^{*}(x, v)=\sup _{u \in \mathbb{R}^{M}}(v \mid u)-F(x, u) .
$$

Let $\epsilon>0$. The perturbed dual action is defined on $(\operatorname{Ker} L)^{\perp}$ by

$$
\Psi_{\epsilon}(v)=\int_{\Omega}\left[\frac{1}{2}(K v \mid v)+F_{\epsilon}^{*}(x, v)\right] d x,
$$

where $F_{\epsilon}^{*}(x, v)$ is the Fenchel transform of $F_{\epsilon}(x, u)=F(x, u)+(\epsilon / 2)|u|^{2}$.

It is assumed that $K$ is the sum of a compact and of a positive definite operator. Because of the non-resonance condition with respect to $\lambda_{1}$, for $\epsilon>0$ small, $\Psi_{\epsilon}$ is coercive on $X=$ $(\operatorname{Ker} L)^{\perp}$ and has a minimizer $v_{\epsilon}$. It suffices then to use the interaction between $F$ and the kernel of $L$ given in (6) to prove a posteriori estimates on $v_{\epsilon}$. Passing to the limit as $\epsilon \downarrow 0$, we obtain a minimizer $v$ of $\Psi$ and, by duality, a solution $u$ of (5). 
Let $\Omega$ be a smooth bounded domain of $\mathbb{R}^{N}$ and let $f: \Omega \times \mathbb{R} \rightarrow \mathbb{R}$ be a Caratheodory function such that

$$
|f(x, u)| \leq a|u|+b
$$

We denote by $\mu_{2}$ the first positive eigenvalue of

$$
\begin{cases}\Delta u+\lambda u=0, & \text { in } \Omega, \\ \frac{\partial u}{\partial n}=0, & \text { on } \partial \Omega .\end{cases}
$$

We assume that

$$
F(x, u)=\int_{0}^{u} f(x, s) d s
$$

satisfies

$$
\varlimsup_{|u| \rightarrow \infty} \frac{2 F(x, u)}{u^{2}} \leq \alpha(x) \leq \mu_{2}
$$

uniformly for, i.e., $x \in \Omega$.

We consider the problem

$$
\begin{cases}\Delta u+f(x, u)=0, & \text { in } \Omega, \\ \frac{\partial u}{\partial n}=0, & \text { on } \partial \Omega .\end{cases}
$$

\section{Theorem 2.1 [17] Assume that}

(a) $f(x, \cdot)$ is nondecreasing for almost all $x \in \Omega$,

(b) $\alpha(x)<\lambda_{1}$ on a subset of $\Omega$ of a positive measure.

Then problem (7) is solvable if and only if there exists $\bar{u} \in \mathbb{R}$ such that

$$
\int_{\Omega} f(x, \bar{u}) d x=0 .
$$

A similar result for the Dirichlet problem

$$
\left\{\begin{array}{l}
u^{\prime \prime}+u+f(x, u)=0, \quad 0<x<\pi, \\
u(0)=u(\pi)=0
\end{array}\right.
$$

is contained in [19].

The general results of [18] are applied to Dirichlet problems, Neumann problems and to periodic solutions of Hamiltonian systems and hyperbolic semilinear equations. In the latter case, the dimension of the kernel of $L$ is infinite. See the survey [21] by Brezis.

It is important to note that the non-resonance assumptions are related to the potential $F(\cdot, x)$, not to the gradient $f(\cdot, x)$. In particular, the Palais-Smale condition is not necessarily satisfied.

General non-resonance conditions are used in [22] in order to prove the existence and uniqueness for semilinear equations in a Hilbert space by variational or iterative methods. Applications are given to semilinear wave equations. 


\section{Two books}

We describe some main features of two books by Jean Mawhin devoted to critical point theory.

The book Problèmes de Dirichlet variationnels non linéaires (1986) is a nice introduction to critical point theory. The main tools,

- minimization,

- dual least action principle,

- minimax methods, and

- Morse theory,

are applied to the simple model problem

$$
\left\{\begin{array}{l}
u^{\prime \prime}=\nabla F(x, u), \quad 0<x<\pi, \\
u(0)=u(\pi)=0 .
\end{array}\right.
$$

A new methodology was used in the construction of Palais-Smale sequences.

Definition 3.1 Let $X$ be a Banach space and let $\Psi \in \mathcal{C}^{1}(X, \mathbb{R})$. A Palais-Smale sequence (at level $c)$ is a sequence $\left(u_{n}\right) \subset X$ such that

$$
\phi^{\prime}\left(u_{n}\right) \rightarrow 0, \quad \phi\left(u_{n}\right) \rightarrow c .
$$

The Palais-Smale condition (at level $c$ ) is satisfied if every Palais-Smale sequence (at level $c$ ) contains a convergent subsequence.

Let us also mention the recent survey [23] on the Palais-Smale condition.

As written in the introduction of [24], the usual minimax method

1. prove an a priori compactness condition, like the Palais-Smale condition,

2. prove a deformation lemma depending upon this condition, and

3. construct a critical value,

could be replaced by the following steps:

1. prove a quantitative deformation lemma,

2. construct a Palais-Smale sequence, and

3. verify a posteriori compactness conditions.

The book [16] contains the first application of this methodology, using the quantitative deformation lemma in [25]. (See [26] for another approach using Ekeland's variational principle in the case of the mountain pass theorem).

The book Critical Point Theory and Hamiltonian Systems (1989) is motivated by the problems

$$
\left\{\begin{array}{l}
\ddot{u}=\nabla F(t, u), \\
u(0)-u(T)=u^{\prime}(0)-u^{\prime}(T)=0
\end{array}\right.
$$

and

$$
\left\{\begin{array}{l}
J \dot{u}=\nabla H(t, u) \\
u(0)=u(T)
\end{array}\right.
$$


Among many other results, a new bifurcation theorem is given. Consider the equation

$$
f(\lambda, u)=0,
$$

where $f(\lambda, u)=\nabla_{u} \phi(\lambda, u)$ and $f(\lambda, 0)=0$. If there is some $n \in \mathbb{N}$ such that the critical groups satisfy

$$
C_{n}(\phi(a, \cdot)) \nsucceq C_{n}(\phi(b, \cdot)),
$$

then there exists a bifurcation point $\left(\lambda_{0}, 0\right) \in[a, b] \times\{0\}$ for $(8)$.

\section{Converse to the Lagrange-Dirichlet theorem}

In 1971, Hagedorn proved that, for Lagrangian systems of class $\mathcal{C}^{2}$, the equilibrium is unstable if it corresponds to a strict local maximum of the potential energy. The proof, using the theory of geodesics on Finsler manifolds, was rather involved. A new proof is given by Hagedorn and Mawhin in [27].

The idea is to replace Jacobi's principle of least action by a new variational principle due to van Groesen [28]. Let $T=p^{T} B(q)^{p}$ be the kinetic energy and let $U=U(q)$ be the potential energy. The functional

$$
J_{h}(q)=\left(\int_{0}^{1} T(q, \dot{q}) d t\right)\left(\int_{0}^{1}(h-U(q)) d t\right)
$$

is minimized on the subset

$$
C=\left\{q \in W^{1,2}(] 0,1[): q(0)=0,\|q(1)\|=c\right\}
$$

of $W^{1,2}(] 0,1[)$ for some suitable $c$.

\section{Neumann problems for the singular $\phi$-Laplacian}

In this section, we describe some recent works motivated by the Neumann problem

$$
\begin{cases}\operatorname{div}\left(\frac{\nabla v}{\sqrt{1-|\nabla v|^{2}}}\right)=g(|x|, v), & \text { in } \Omega, \\ \frac{\partial v}{\partial n}=0, & \text { on } \partial \Omega\end{cases}
$$

where

$$
\Omega=\left\{x \in \mathbb{R}^{N}: r_{1} \leq|x| \leq r_{2}\right\} \quad\left(0 \leq r_{1}<r_{2}<\infty\right) .
$$

The general problem treated in $[12,29]$ and $[30]$ is

$$
\left\{\begin{array}{l}
{\left[r^{N-1} \phi\left(u^{\prime}\right)\right]^{\prime}=r^{N-1} f(r, u)} \\
u^{\prime}\left(r_{1}\right)=u^{\prime}\left(r_{2}\right)=0
\end{array}\right.
$$


The function $\phi=\Phi^{\prime}$ satisfies assumption (4). Let us define

$$
\begin{aligned}
& X=W^{1, \infty}(] r_{1}, r_{2}[), \\
& C=\left\{u \in X:\left\|u^{\prime}\right\|_{\infty} \leq a\right\}, \\
& K(u)= \begin{cases}\int_{R_{1}}^{R_{2}} \Phi\left(u^{\prime}\right) r^{N-1} d r, & u \in C, \\
+\infty, & u \in X \backslash C,\end{cases} \\
& J(u)=\int_{r_{1}}^{r_{2}} G(r, u) r^{N-1} d r, \quad u \in X,
\end{aligned}
$$

where $G(r, u)=\int_{0}^{u} g(r, s) d s$.

Then Szulkin's critical point theory [11] is applicable to $I=J+K$, since $K$ is a convex l.s.c. function and since $J$ is a differentiable function. The strategy is to prove that a critical point of $I$ in the sense of Definition 1.3 satisfies $\left\|u^{\prime}\right\|_{\infty}<a$ and hence is a solution of (9).

Assume, for example, that

$$
|g(r, x)| \leq f(r) \in L^{1}(] r_{1}, r_{2}\left[, r^{N-1} d r\right) .
$$

Then (9) is solvable if

$$
\lim _{|x| \rightarrow \infty} \int_{r_{1}}^{r_{2}} G(r, x) r^{N-1} d r=+\infty
$$

or if

$$
\lim _{|x| \rightarrow \infty} \int_{r_{1}}^{r_{2}} G(r, x) r^{N-1} d r=-\infty .
$$

The first case corresponds to a ground state of $I$ and the second case to a saddle point of $I$ (see [12]). The case of mountain pass solutions is also treated. The generalization of those results to the non-radial case is a challenging open problem.

\section{Competing interests}

The author declares that he has no competing interests.

\section{Received: 29 January 2013 Accepted: 22 April 2013 Published: 7 May 2013}

\section{References}

1. Mawhin, J: Periodic oscillations of forced pendulum-like equations. In: Ordinary and Partial Differential Equations. Lecture Notes in Math., vol. 964, pp. 458-476. Springer, Berlin (1982)

2. Mawhin, J: Periodic solutions of second order nonlinear difference systems with $\phi$-Laplacian: a variational approach. Nonlinear Anal. 75(12), 4672-4687 (2012)

3. Mawhin, J: Global results for the forced pendulum equation. In: Handbook of Differential Equations, pp. 533-589. North Holland, Amsterdam (2004)

4. Mawhin, J, Willem, M: Multiple solutions of the periodic boundary value problem for some forced pendulum-type equations. J. Differ. Equ. 52(2), 264-287 (1984)

5. Mawhin, J: Forced second order conservative systems with periodic nonlinearity. Ann. Inst. Henri Poincaré, Anal. Non Linéaire 6, 415-434 (1989)

6. Franks, J: Generalizations of the Poincaré-Birkhoff theorem. Ann. Math. 128(2), 139-151 (1988)

7. Franks, J: Erratum to: 'Generalizations of the Poincaré-Birkhoff theorem'. Ann. Math. 164(2), 1097-1098 (2006)

8. Fučík, S: Solvability of Nonlinear Equations and Boundary Value Problems. Mathematics and Its Applications. Reidel, Dordrecht (1980)

9. Mawhin, J: Periodic solutions of the forced pendulum: classical vs relativistic. Matematiche 65(2), 97-107 (2010)

10. Brézis, H, Mawhin, J: Periodic solutions of the forced relativistic pendulum. Differ. Integral Equ. 23(9-10), 801-810 (2010) 
11. Szulkin, A: Minimax principles for lower semicontinuous functions and applications to nonlinear boundary value problems. Ann. Inst. Henri Poincaré, Anal. Non Linéaire 3(2), 77-109 (1986)

12. Bereanu, C, Jebelean, P, Mawhin, J: Variational methods for nonlinear perturbations of singular $\varphi$-Laplacians. Atti Accad. Naz. Lincei Cl. Sci. Fis. Mat. Natur. Rend. Lincei (9) Mat. Appl. 22, 89-111 (2011)

13. Brézis, H, Mawhin, J: Periodic solutions of Lagrangian systems of relativistic oscillators. Commun. Appl. Anal. 5(2-4), 235-250 (2011)

14. Clarke, F: Solution périodique des équations hamiltoniennes. C. R. Acad. Sci. Paris Sér. A-B 287(14), A951-A952 (1978)

15. Clarke, F, Ekeland, I: Solutions périodiques de période donnée, des équations hamiltoniennes. C. R. Acad. Sci. Paris Sér. A-B 287(15), A1013-A1015 (1978)

16. Mawhin, J: Problèmes de Dirichlet variationnels non linéaires. Séminaire de Mathématiques Supérieurs. Presses de I'Université de Montréal, Montreal (1987)

17. Mawhin, J, Ward, JR Jr., Willem, M: Variational methods and semilinear elliptic equations. Arch. Ration. Mech. Anal. 95(3), 269-277 (1986)

18. Mawhin, J, Willem, M: Critical points of convex perturbations of some indefinite quadratic forms and semi-linear boundary value problems at resonance. Ann. Inst. Henri Poincaré, Anal. Non Linéaire 3(6)، 431-453 (1986)

19. Mawhin, J, Ward, JR Jr., Willem, M: Necessary and sufficient conditions for the solvability of a nonlinear two-point boundary value problem. Proc. Am. Math. Soc. 93(4), 667-674 (1985)

20. Mawhin, J, Willem, M: Critical Point Theory and Hamiltonian Systems. Aplied Mathematical Sciences, vol. 74. Springer, New York (1989)

21. Brézis, H: Periodic solutions of nonlinear vibrating strings and duality principles. Bull. Am. Math. Soc. 8(3), 409-426 (1983)

22. Fonda, A, Mawhin, J: Iterative and variational methods for the solvability of some semilinear equations in Hilbert spaces. J. Differ. Equ. 98(2), 355-375 (1992)

23. Mawhin, J, Willem, M: Origin and evolution of the Palais-Smale condition in critical point theory. J. Fixed Point Theory Appl. 7(2), 265-290 (2010)

24. Willem, M: Minimax Theorems. Progress in Nonlinear Differential Equations and Their Applications, vol. 24. Birkhäuser Boston, Boston (1996)

25. Willem, M: Lectures on Critical Point Theory. Trabalho de matemática. Universidade de Brasília, Brasília (1983)

26. Aubin, JP, Ekeland, I: Applied Nonlinear Analysis. Pure and Applied Mathematics. Wiley, New York (1984)

27. Hagedorn, P, Mawhin, J: A simple variational approach to a converse of the Lagrange-Dirichlet theorem. Arch. Ration. Mech. Anal. 120(4), 327-335 (1992)

28. van Groesen, E: Analytical mini-max methods for Hamiltonian brake orbits of prescribed energy. J. Math. Anal. Appl. $132,1-12(1988)$

29. Bereanu, C, Jebelean, P Mawhin, J: Multiple solutions for Neumann and periodic problems with singular $\phi$-Laplacian. J. Funct. Anal. 261(11), 3226-3246 (2011)

30. Mawhin, J: Radial solutions of Neumann problem for periodic perturbations of the mean extrinsic curvature operator Milan J. Math. 79, 95-112 (2011)

doi:10.1186/1687-2770-2013-115

Cite this article as: Willem: Jean Mawhin's contributions to critical point theory. Boundary Value Problems 2013

2013:115.

\section{Submit your manuscript to a SpringerOpen ${ }^{\ominus}$ journal and benefit from:}

- Convenient online submission

- Rigorous peer review

- Immediate publication on acceptance

- Open access: articles freely available online

- High visibility within the field

- Retaining the copyright to your article 